\title{
Design and field evaluation of hill-drop pneumatic central cylinder direct-seeding machine for hybrid rice
}

\author{
Baolong Wang, Xiwen Luo*, Zaiman Wang, Le Zheng, Minghua Zhang, Yizheng Dai, He Xing \\ (Key Laboratory of Key Technology on Agricultural Machine and Equipment, Ministry of Education, \\ South China Agricultural University, Guangzhou 510642, China)
}

\begin{abstract}
To meet the requirement of the precision direct-seeding for hybrid rice, this study aimed to design a hill-drop pneumatic central cylinder direct-seeding machine to sow ten rows at a time. A series of orthogonal experiments were conducted to investigate the performance of the cylinder seeder. The influences of the hole diameters, degree of vacuum, and rotational speed of the cylinder were tested on JPS-12 computer-vision seeding test platform, and the rotational speed of 10-50 $\mathrm{r} / \mathrm{min}$, diameter of $135 \mathrm{~mm}$ and a negative pressure of 1.0-2.0 $\mathrm{kPa}$ were employed. Test results showed that the optimal parameter combination was a vacuum of $2.0 \mathrm{kPa}$ and a hole diameter of $2.0 \mathrm{~mm}$ (straight hole), with a rotational speed of $30 \mathrm{r} / \mathrm{min}$. The probability of $(2 \pm 1)$ seeds in each hole was $95.3 \%$, while the probability of seed-missing hole was $2.0 \%$. A series of field experiments were then conducted to test the seeder performance according to China National Standard Test Methods, and the field test results showed that for the hill-drop pneumatic central cylinder direct-seeding machine, the probability of $(2 \pm 1)$ seeds in each hill was $91.6 \%$, while the probability of seed-missing hill was $2.7 \%$. The yield data showed that the average effective panicle had 231.25 ears, the average seed setting rate was $9.92 \%$, the average 1000 -grain weight was $22.45 \mathrm{~g}$, and the average yield was $7107.9 \mathrm{~kg} / \mathrm{hm}^{2}$ that was $26.14 \%$ higher than the average yield of rice $\left(5634.9 \mathrm{~kg} / \mathrm{hm}^{2}\right)$ of Guangdong Province in 2016. The results showed that the hill-drop pneumatic central cylinder direct-seeding machine for hybrid rice could be applied in practical precision rice seeding.
\end{abstract}

Keywords: direct-seeding machine, hybrid rice, precision rice seeder, pneumatic cylinder, platform test, field test DOI: $10.25165 /$ j.ijabe.20181106.4175

Citation: Wang B L, Luo X W, Wang Z M, Zheng L, Zhang M H, Dai Y Z, et al. Design and field evaluation of hill-drop pneumatic central cylinder direct-seeding machine for hybrid rice. Int J Agric \& Biol Eng, 2018; 11(6): 33-40.

\section{Introduction}

Rice is one of the most important food crops in the world. More than half of the world's population relies on rice as a staple food. Rice is planted in 122 countries, and the annual area of cultivation is $140-157$ million $\mathrm{hm}^{2}$, with $90 \%$ of the planted area being concentrated in Asia. Rice is also one of the most important cultivated crops in China. In 2015, the total planted area was 30.2 million $\mathrm{hm}^{2}$, making great contribution to the growing global demand of food ${ }^{[1,2]}$.

Hybrid rice, which offers a strong tilling ability and high yield, has been popularized and widely researched in China. In 2013, 16.18 million $\mathrm{hm}^{2}$ were planted with hybrid rice, accounting for $53.37 \%$ of the overall rice-planted area ${ }^{[3]}$.

The mechanization of rice planting is the most difficult aspect in the rice production. Precision rice hill drop drilling offers the

Received date: 2018-02-09 Accepted date: 2018-10-22

Biographies: Baolong Wang, $\mathrm{PhD}$ candidate, research interests: agricultural mechanization and automation, Email: 1531203262@qq.com; Zaiman Wang, $\mathrm{PhD}$, Associate Researcher, research interests: agricultural mechanization and automation, Email: wangzaiman@scau.edu.cn; Le Zheng, PhD, research interests: agricultural mechanization and automation, Email: 451016523@ qq.com; Minghua Zhang, PhD, Postdoctor, research interests: agricultural mechanization and automation, Email: zhangminghuascau@163.com; Yizheng Dai, $\mathrm{PhD}$, research interests: agricultural mechanization and automation, Email: nc_vip@163.com; He Xing, PhD candidate, research interests: agricultural mechanization and automation, Email: 675974347@qq.com.

*Corresponding author: Xiwen Luo, Professor, research interests: agricultural mechanization and automation. 483 Wushan Road, Tianhe District, Guangzhou, 510642, China. Tel/Fax: +86-20-38676975, Email: xwluo@ scau.edu.cn. advantages of saving labor and time, ensuring good ventilation, reducing the risks of pests and diseases, providing a low tiller node, and promoting good long-term growth. The precision rice hill-drop drilling machine developed by Xiwen Luo of the South China Agricultural University has been widely used in more than 20 provinces in China and other countries such as Laos, Cambodia, and Thailand ${ }^{[4,5]}$. However, it was optimized mainly for conventional rice and it offered a precision of 3-8 seeds per hill. Due to the mechanical structure of combined hole-type metering device, it is difficult to satisfy the few precision sowing requirements of hybrid rice direct-seeding ${ }^{[6-8]}$.

According to the growth characteristics of hybrid rice and the demand for few precision hill-drop drilling, the pneumatic seed-metering seeder have been found to offer a better alternative. Pneumatic seeding devices inflict less damage to a sprouting seed, and are less demanding of the shape and size of the seed. Zhang et al ${ }^{[9,10]}$ and Xing et al. ${ }^{[11,12]}$ designed and optimized a vertical disk-type pneumatic seeder which was capable of the precision sowing of hybrid rice, but the structure is complex. Zhang et al. ${ }^{[13]}$ designed a pneumatic cylinder seeding device for rice, which involved hole filling, multi-hole suction, air blowing, seed belt protection, and pneumatic blockage clearing. However, the precision seeding performance requires further improvement. Fu et al. $^{[14]}$ designed a side-ventilated seed-metering device, and performed single-factor and quadrature tests on its suction, negative pressure, blow flow rate, and operating speed in the laboratory using round vegetable seeds. Zhao et al. ${ }^{[15]}$ designed a seed-metering device with an internal pressure diaphragm plate, optimized the parameters of the diameter and shape of the suction hole as well as the rotational speed, and analyzed the kinetics of the 
suction process, but it was only for small seeds. Zuo et al. ${ }^{[16,17]}$ designed a hole-type and narrow-gap air suction seeding cylinder, and examined the function in commercial-scale seedling production of hybrid rice. Gao et al. ${ }^{[18]}$ designed an air suction cylinder seed-metering device for so-called "pseudo-ginseng" to determine its main structural parameters, and also devised a seed-filling and investment process mechanics model, conducted investment comparison tests to verify the necessity for zero-speed investment, as applied to the field shed. Liao et al. ${ }^{[19]}$ designed a novel prototype of pneumatic precision centralized seed-metering device with seed inside-filling for rapeseed. It can sow six rows at a time. Tests were conducted to optimize the working parameters. Yu et al. ${ }^{[20]}$ studied the influence on the performance of pneumatic precision metering device for rapeseed with positive and negative pressures, the simulation analysis and match experiment was carried to optimize the performance of the seeder. Karayel et al. ${ }^{[21]}$ studied and obtained the regression equation linking the vacuum degree of the gas chamber, the quality of 1000 seeds, the cross-sectional area of the suction hole, the seed sphericity, and the seed density. Singh et al. ${ }^{[22]}$ optimized the structure of pneumatic cotton seed-metering device. The design featured a pneumatic cylinder seed-metering device that continuously rotated during suction and seeding to achieve high-efficiency seeding characteristics. This design has become one of the main types of pneumatic seeding device.

There has been many scientific research for the design of cylinder type seed metering seeder ${ }^{[23-28]}$, but few studies addressed the direct-seeding of hybrid rice. According to the hybrid rice agronomy seeding characteristics about few precision sowing of the precision hill-drop drilling, the present study was to design a hill-drop pneumatic central cylinder direct-seeding machine for hybrid rice. The rotational speed, air pressure, and hole type of pneumatic central cylinder were studied, while the effects of the optimal mathematical model were optimized. Platform and field tests for determination of the seeding performance were carried out, which provided the base for the further optimization of the hill-drop pneumatic central cylinder direct-seeding machine for hybrid rice.

\section{Overall structure and operating principle}

\subsection{Machine structure and technical parameters}

The hill-drop pneumatic central cylinder direct-seeding machine for hybrid rice includes the Yanmar VP-6D riding rice transplanter head, vortex pump, seed box, lateral sucking mechanism, seed cylinder mount, guide tubes, ditching and ridging chassis, and other components. The pneumatic central-cylinder seeder connects to a mount, which hinged onto the ditching and ridging chassis.

The ditching and ridging chassis consists of a sowing and water ditch opener. The chassis hinged with the riding rice transplanter head through a three-point hanging mechanism as shown in Figure 1, which consists of an upper pull rod and two lower rods. There are two floating boxes between the drive and the base, which float according to the conditions presented by the paddy surface and controlled through the hydraulic system and the three-point suspension ${ }^{[4]}$.
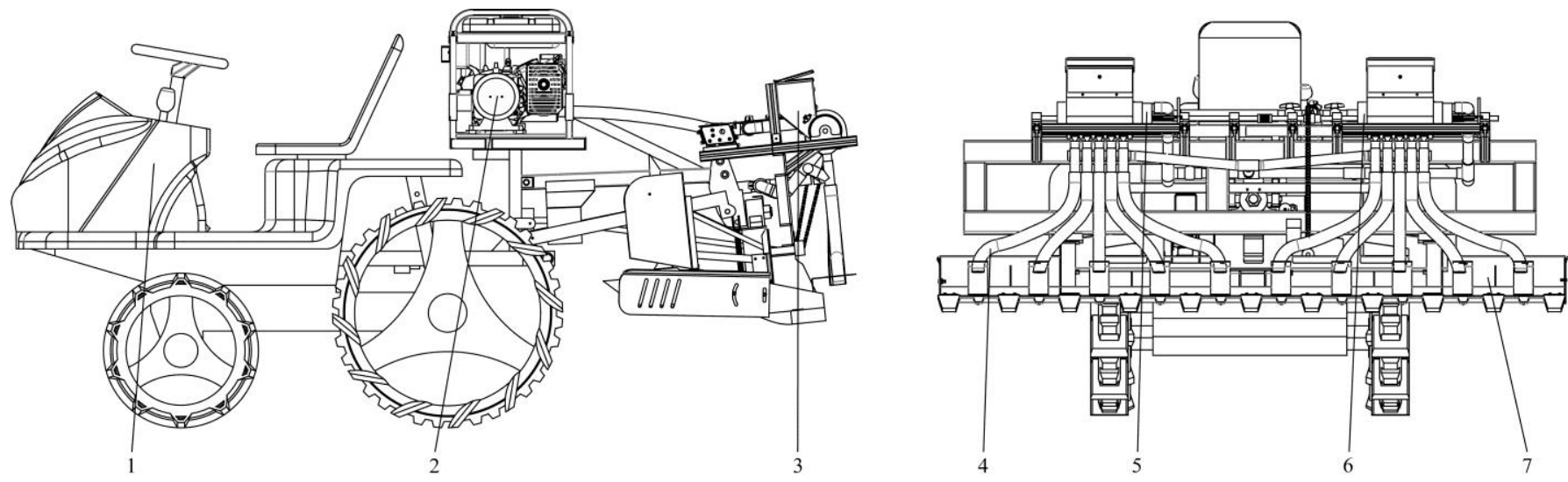

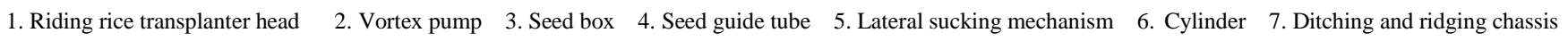

Figure 1 Configuration of hill-drop pneumatic central cylinder direct-seeding machine for hybrid rice

The power source for the direct seeder is a Yanmar VP-6D Yanmar riding rice transplanter. According to the number of seeding seeder and the number of holes in the cylinder, the ratio of the power take-off (PTO) to the rotational speed of the cylinder is 9:1 to ensure a certain ratio between the machine speed and rotational speed of the cylinder, and ensure the stability of the hill spacing.

The relationship between the rotational speed of the cylinder and the machine speed is defined as ${ }^{[29]}$ :

$$
n=6000 \frac{v}{K l}
$$

where, $n$ is the rotational speed of the cylinder, $\mathrm{r} / \mathrm{min} ; v$ is forward speed of the machine, $\mathrm{m} / \mathrm{s} ; K$ is the number of circumferential holes, and $l$ is the hill spacing, $\mathrm{m}$.

According to Equation (1), for a given machine speed, the hill spacing is inversely proportional to the rotational speed of the cylinder. For a given hill spacing, the speed of the seeding machine is inversely proportional to the rotational speed of the cylinder.

The main parameters for the overall machine are listed in Table 1.

Table 1 Main machine parameters

\begin{tabular}{lc}
\hline \multicolumn{1}{c}{ Item } & Parameter \\
\hline Power source & Yanmar VP-6D $(13.2 \mathrm{~kW})$ \\
Linkage & Three-point \\
Seeding rows & 10 \\
Seeder number & 2 \\
Seeder type & Cylinder \\
Dimensions $(\mathrm{L} \times \mathrm{H} \times \mathrm{W}) / \mathrm{m}^{3}$ & $1.5 \times 2.6 \times 1.7$ \\
Operating speed $/ \mathrm{m} \cdot \mathrm{s}^{-1}$ & $0-1.5$ \\
\hline
\end{tabular}

\subsection{Operating principle}

The operating of the hill-drop pneumatic central cylinder direct-seeding machine for hybrid rice can be divided into four 
stages: seed sucking, seed carrying, seed discharging and seed blowing. While the machine is operating, the seed appears to be "boiling" in the box, which is the result of the action of the electromagnetic exciter. The cylinder is driven by the PTO of the transplanter, and the hole on the cylinder sucked up the seed in the box by applying negative pressure. The seed conveyed to the seed discharge position by the rotation of the cylinder, and then entered the seed-blowing pipeline, deposited into the seeding ditch under the influence of the positive airflow and gravity.

\section{Design of pneumatic-cylinder distributor device}

\subsection{Overall configuration}

The seed-metering device is one of the key components to achieve the precision seeding of hybrid rice. Traditional mechanical seeder is not capable of few precision sowing requirement for hybrid rice. Thus, a hole-based design and air-flow control for pneumatic central cylinder seeding device was developed in this study to achieve the desired seeding amount. The main components of a pneumatic central-cylinder seeding device were shown in Figure 2, include seed box (1), ventilation chamber shell (2), seeding cylinder (3), and the seed guide tubes (4).

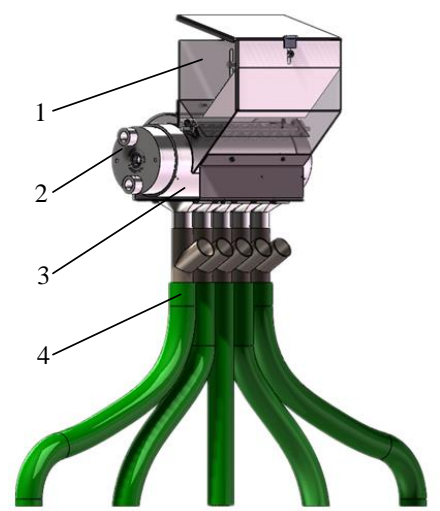

1. Seed box 2..Ventilation chamber shell 3. Cylinder 4. Guide tube

Figure 2 Configuration of pneumatic seed-metering device

\subsection{Double-cylinder}

The outer cylinder 1 and inner cylinder 3 nested together as shown in Figure 3. The ventilation chamber shell 7 is installed coaxially with the cylinders, with a lateral spring and screw rod that in movable fit. The ventilation chamber shell 7 is divided into a negative-pressure zone 5 and a positive-pressure zone 6 . A row of suction holes 2 in the negative pressure zone 5 sucks the seeds out of the seed box and excess seeds are removed by the cleaning mechanism. The sucked seeds are carried into the positive-pressure zone 6 by the rotation of the cylinder, after which the seeds drop into the pipeline under the influence of gravity and the positive pressure. The seeds are then deposited into the seeding furrow by airflow.

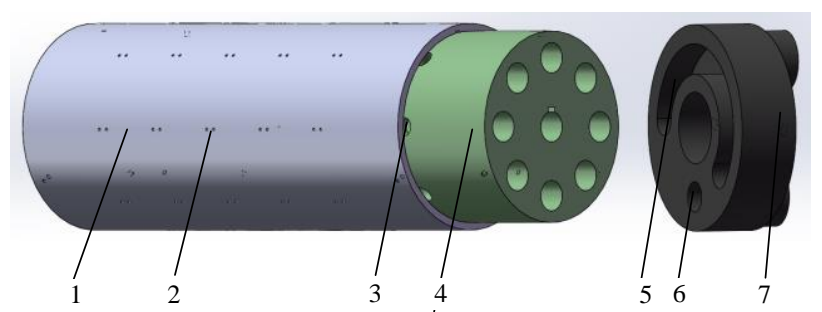

1. Outer cylinder 2. Outer suction holes 3. Inner cylinder 4. Inner suction holes 5. Negative-pressure zone 6. Positive-pressure zone 7. Ventilation chamber shell

Figure 3 Cylinders and ventilation chamber shell
The rotational speed of the cylinder is the design basis for the cylinder diameter. There is a given numerical relationship between the operating principle of the seed-metering cylinder and the machine ${ }^{[29]}$.

The amount of seed quantity by the cylinder per second is given by:

$$
Q_{m}=\frac{V_{g}}{D+\Delta L}
$$

It also can be expressed in terms of the forward speed $V_{m}$ and the hill space $T$ as:

$$
Q_{m}=\frac{V_{m}}{T}
$$

Eliminating $Q_{m}$ from Equations (2) and (3) gives:

$$
V_{g}=\frac{V_{m}(D+\Delta L)}{T}
$$

The cylinder diameter can be calculated from:

$$
D_{g}=\frac{60 V_{m}(D+L)}{\pi N T}
$$

The number of suction holes can be calculated from:

$$
Z=\frac{\pi D_{g} K}{D+\Delta L}
$$

where, $Q_{m}$, The Amount of seed quantity per second; $V_{g}$, Cylinder linear velocity, $\mathrm{m} / \mathrm{s} ; D$, Suction hole diameter, $\mathrm{mm} ; \Delta L$, Arc length between suction holes, mm; $V_{m}$, Forward speed, $\mathrm{m} / \mathrm{s} ; T$, Sowing spacing, $\mathrm{mm} ; D_{g}$, Cylinder diameter, $\mathrm{mm} ; N$, Rotational speed of cylinder, r/min; $Z$, No. of suction holes; $K$, No. of suction hole rows.

Thus, $D+\Delta L$ is the arc length between adjacent suction holes, which must be greater than the maximum size of two rice seeds. This can be calculated as:

$$
D+\Delta L \geq 2 l \max
$$

From Equation (3), when the machine speed $V_{m}$ and the sowing spacing of the seeder is known, the diameter of the cylinder will be inversely proportional to the rotational speed. When the rotational speed is decreased, the seed fill time can be increased, but the cylinder large diameter can lead to an increase in the required airflow, and therefore create greater air leakage. However, the use of a smaller cylinder would reduce the seed fill time and the seed height in the seed box. The cylinder fabrication material should also be taken into account.

According to the agronomy characteristics of precision rice hill drop direct seeding and practical experience, the speed of a direct-seeding machine in a paddy field is in the range of 0.5 $1.5 \mathrm{~m} / \mathrm{s}$, for the arc length between adjacent suction holes $D+\Delta L \geq 0.02 \mathrm{~m}$. Thus, given the PTO rotational speed and the transmission ratio of the gearbox, the rotational speed of the cylinder is $0-60 \mathrm{r} / \mathrm{min}$. The sowing spacing is generally $0.14-0.18$ $\mathrm{m}$ for hybrid rice, and the cylinder diameter can be estimated from Equation (5). Considering the existing transmission systems of direct-seeding machines and other factors ${ }^{[4,5]}$, the ideal outer diameter is $135 \mathrm{~mm}$.

Assuming the above cylinder configuration, that is, an outer cylinder diameter of $135 \mathrm{~mm}$ with a thickness of $5 \mathrm{~mm}$, the inner cylinder diameter will be $125 \mathrm{~mm}$. Both cylinders are fabricated by nylon. The holes in the inner cylinder are $8 \mathrm{~mm}$ in diameter, while the axial round grooves are $20 \mathrm{~mm}$ in diameter. These are arranged into rings of 8 grooves.

The double structure of the cylinder means that the outer cylinder can be replaced ${ }^{[28]}$, with a slightly different cylinder being used according to the rice variety and seeding requirement. 


\subsection{Churning and Cleaning device}

Based on the analysis of the churning devices influence on seed absorption performance with the vertical disk ${ }^{[9,10]}$, a wedge type servo stirring mechanism for cylinder is designed. The inner and outer surfaces are all arc and installed on cylinder surface. Its minimum thickness is $1 \mathrm{~mm}$, the maximum thickness is $2 \mathrm{~mm}$, the diameter near the outer wall of the roller is $135 \mathrm{~mm}$ diameter, and the straight distance between the two ends is $4.75 \mathrm{~mm}$. Its structure is shown in Figure 5a. A churning mechanism is arranged between the two holes, and the circumferential distribution is 8 , a total of five rows. The distribution is shown in Figure 5a.

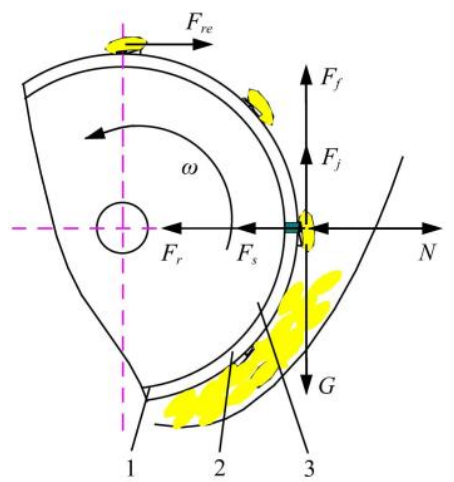

1. Outer cylinder 2. Outer suction holes 3 . Inner cylinder

Figure 4 Mechanical analysis of adsorbed seed

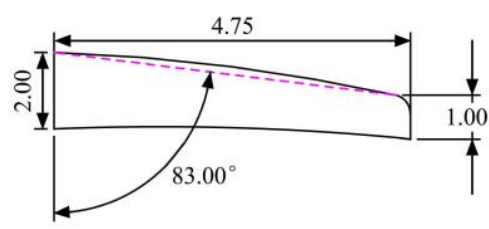

a. Churning devices

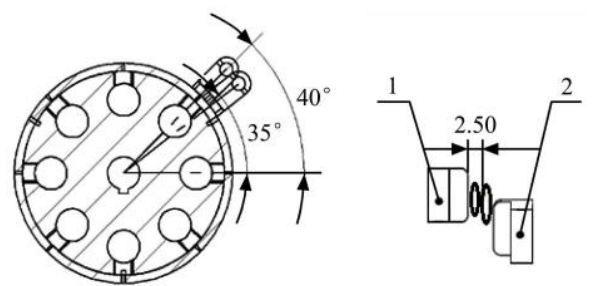

1. Left cleaning mechanism 2. Right cleaning mechanism b. Cleaning mechanism
As shown in Figure 4, the force analysis of the rice seed on the cylinder is carried out. The gravity $(G, N)$, the centrifugal force $\left(F_{r}, N\right)$, the friction force $\left(F_{f}, N\right)$, the air suction $\left(F_{s}, N\right)$ are synthesized by the synthetic vector method, the resistance force $\left(F_{j}, N\right)$ from the cleaning devices and the resultant force is $Q$ which can be expressed as:

$$
Q=G+F_{r}+F_{f}+F_{s}+F_{j}
$$

For a mechanical seed-metering device, it is necessary to remove excess seeds from the sucking holes by using a cleaning device. The cleaning devices are divided into mechanical and airflow types.

Working principle analysis: In the present study, a mechanical bilateral-arc cleaning device was adopted to remove excess rice seeds adsorbed onto both sides of the suction holes. The cleaning devices are installed on the fixed axis. The excess seeds are cleaned away as follows: as the Equation (8) mentioned, and the resistance force $F_{j}$ from the cleaning devices overcome the $F_{f}$, the excess seeds (more than one with the single hole) were cleaned and returned to the seed box. According to the seed amount required for each hill and the friction between the cylinder and seeds ${ }^{[30]}$ : two cleaning devices are provided and installed at angles of $40^{\circ}$ and $35^{\circ}$. In order to cleaning the excess seeds, the distance from the cleaning device edge to the hole is $2.50 \mathrm{~mm}$ (longer than the width of one seed, to ensure one suction including one seed). The layout is shown in Figures $5 \mathrm{~b}$ and $5 \mathrm{c}$.

Figure 5 Sketch map of cleaning mechanism and churning devices

\subsection{Crossed-diversion seed box}

The fluidity of rice seed is very low and the excessive accumulation of seeds on the cylinder can greatly affect the seed suction and cleaning.

A cross-diversion device installed in the seed box is shown in Figure 6. The two guide plates can be moved up and down. According to the friction characteristics between the rice seeds and the steel plates ${ }^{[31]}$, the distance between D1 and D2 can increase the fluidity of the rice. The first outlet and second outlet should be greater than $20 \mathrm{~mm}$ and $25 \mathrm{~mm}$, respectively. The angle between the main diversion plate 1 and the assist diversion plate 3 is $45^{\circ}$, relative to the horizontal ${ }^{[32]}$.

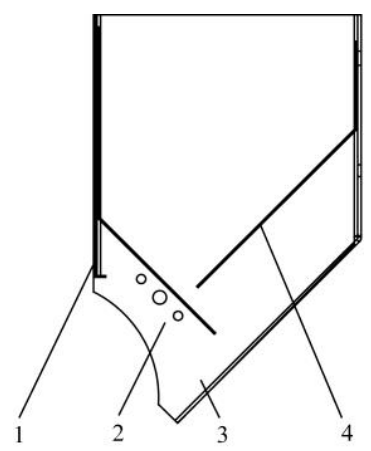

1. Main diversion board 2. First outlet 3. Second outlet 4. Assist diversion board Figure 6 Crossed-diversion seed box mechanism

\section{Selection of vortex pump and valve system}

\subsection{Seed suction conditions and vortex pump selection}

The air source and piping system are necessary to enable the operation of a pneumatic seeder, with the vortex pump air flow and the piping system have major influence on the seed suction performance. A mechanical analysis is as follows ${ }^{[33]}$ :

The airflow suction pressure acting on the seeds is:

$$
P=\frac{1}{2} C p S v^{2}
$$

The combined force of gravity and friction acting on the seed is:

$$
F=P_{y} \tan \theta
$$

where, $C$ is a dimensionless coefficient; $p$ is the air density; $S$ is the area of the seed in a plane perpendicular to the direction of motion, $\mathrm{m}^{2} ; v$ is the velocity of the air flow, $\mathrm{m} / \mathrm{s} ; P_{y}$ is the circumferential pressure of the seed, $\mathrm{kPa} ; \theta$ is the internal friction angle, $\left({ }^{\circ}\right)$.

Seed suction condition:

$$
\frac{1}{2} C p S v^{2}>\mathrm{mg}+P_{y} \tan \theta
$$

When the hole diameter is $0.8-3.0 \mathrm{~mm}, \alpha=0.17-0.72$. The required air flow is:

$$
Q=k_{x} v S_{1} n N
$$

where, $k_{x}$ is the suction coefficient; $n$ is the number of vacuum zone suction holes; and $N$ is the seeder number. 
The suction coefficient $k_{x}$ is the ratio between the velocity of holes that have sucked up seeds and those that have not sucked up seeds:

$$
K_{x}=V_{c} / V
$$

Tests have shown that, for a pressure of $2 \mathrm{kPa}$, the required air velocity is $10.2 \mathrm{~m} / \mathrm{s}$, for a direct-seeding machine with two seeder units and a seeding tube diameter of $36 \mathrm{~mm}$, the total required instantaneous volume flow, $Q$, is given by Equation (13):

$$
Q=2 S V
$$

where, $Q$ is the instantaneous volume flow, $\mathrm{m}^{3} / \mathrm{h} ; S$ is the pipe cross-sectional area, $\mathrm{m}^{2}$, and $V$ is the air-flow velocity, $\mathrm{m} / \mathrm{s}$.

Therefore, using the above equations, the working airflow is determined to be $124.52 \mathrm{~m}^{3} / \mathrm{h}$. Due to the complex operating conditions such as the vibrations during operation, the processing error of parts and other factors, the actual working flow should be greater than the test requirements.

The limited power of the riding rice transplanter $(13.24 \mathrm{~kW})$ makes it unsuitable to drive the vortex pump, and an integrated gasoline-engine vortex pump (Honda GX160 manual generator $(4.06 \mathrm{~kW})$ is adopted. This single-cylinder, four-stroke unit as shown in Figure 7, has a flow of $200 \mathrm{~m}^{3} / \mathrm{h}$, with a size of $304 \mathrm{~mm} \times$ $362 \mathrm{~mm} \times 335 \mathrm{~mm}(L \cdot W \cdot H)$ and a weight of $16.0 \mathrm{~kg}$. The maximum pressure is $25 \mathrm{kPa}$.

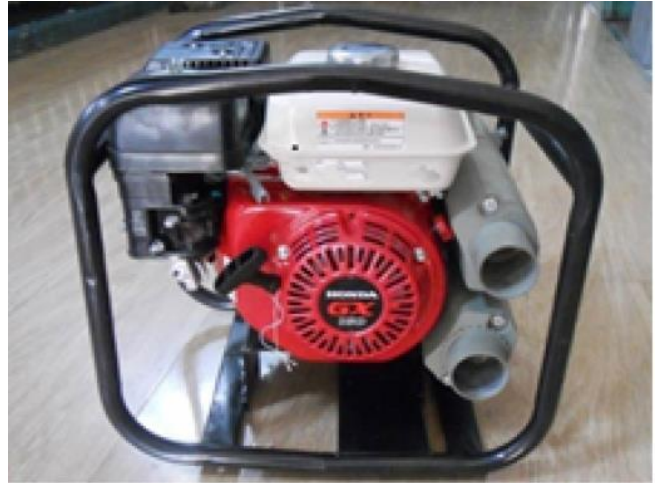

Figure 7 Integrated gasoline-engine vortex pump

\subsection{Seeding pipe flow uniformity test}

The pipe flow is the key for uniform sowing. In present study, the uniformity of the airflow in the distribution pipelines was examined.

For the test, the gasoline vortex pump throttle was demarcated into three zones, namely low, medium, and high. An anemometer was used at the outlets of the pipelines to measure the airflow. Measurements were taken in triplicate at each of the throttle settings. Results were recorded according to the coefficients and the experimental setup, and were listed in Table 2.

\begin{tabular}{|c|c|c|c|c|c|c|c|c|c|c|c|c|}
\hline \multirow{2}{*}{ Gas pedal } & \multicolumn{11}{|c|}{ Air flow at pipeline port $/ \mathrm{m} \cdot \mathrm{s}^{-1}$} & \multirow{2}{*}{ Coefficient of variation $/ \%$} \\
\hline & 1 & 2 & 3 & 4 & 5 & 6 & 7 & 8 & 9 & 10 & Average & \\
\hline Low & 1.572 & 1.687 & 1.644 & 1.486 & 1.464 & 1.826 & 1.623 & 1.639 & 1.795 & 1.415 & 1.635 & 8.0 \\
\hline Medium & 1.514 & 1.764 & 1.836 & 1.502 & 1.653 & 1.952 & 1.565 & 1.562 & 1.716 & 1.665 & 1.673 & 8.8 \\
\hline High & 3.185 & 3.469 & 3.276 & 2.997 & 2.818 & 3.625 & 3.031 & 3.018 & 4.021 & 3.000 & 3.194 & 8.4 \\
\hline
\end{tabular}

Table 2 Results of air flow measurements at pipe outlets

Test results show that the uniform coefficient of variation $(\mathrm{CV})$ for the different air flows at the outlets of the ten-row seed pipes is less than $10 \%$ for the three different degrees of throttle opening of the gasoline vortex pump.

\section{Seeder performance test}

\subsection{Test design}

Given the demand for a means of direct seeding hybrid rice, this study proposes a comprehensive evaluation index standard as follows: $(2 \pm 1)$ particles per hill is qualified, 0 particles per hill is missing, $\geq 4$ particles per hill is the reseeding rate. According to national standard GB-T6973-2005 ${ }^{[34]}$, the number of sucked seeds in each hill was continuously recorded for 250 holes, repeated three times, and the average seeding amount $X$ was determined using Equation (14). The sucked number for each hole was calculated as: $0,1,2,3,4,5 \ldots \ldots$; then the occurrence frequency of the number of seeds sucked is given by Equation (15):

$$
\begin{gathered}
\bar{X}=\frac{1}{750} \sum_{J}^{3}\left(\sum_{i}^{250} X_{k}\right) \\
k=1-250, j=1,2,3 \\
P(i)=\frac{\sum_{i=1}^{n} x_{k j}}{750} \\
K=0,1,2,3,4,5,6 \cdots ; j=1,2,3
\end{gathered}
$$

According to the results of pre-tests, the frequency of the electromagnetic vibrator was set to $60 \mathrm{~Hz}$. The speed of the seed-metering device (A), the vacuum degree (B), and the parameter of the hole $(\mathrm{C})$ were taken as experimental factors. The seeder performance was determined by $\mathrm{L}_{25}\left(5^{6}\right)$ orthogonal testing ${ }^{[34,35]}$. The test factors are listed in Table 3 , the experimental plan and test results are listed in Table 4, the intuitive analysis are listed in Table 5 .

Table 3 Experimental variables and levels

\begin{tabular}{cccc}
\hline & \multicolumn{4}{c}{ Factors } \\
\cline { 2 - 4 } Level & A. Rotational speed $/ \mathrm{r} \cdot \mathrm{min}^{-1}$ & B. Vacuum/kPa & C. Hole type/mm \\
\hline 1 & 10 & 1.0 & 1.5 \\
2 & 20 & 1.5 & 2.0 \\
3 & 30 & 2.0 & 2.0 (Chamfering fillet) \\
4 & 40 & & \\
5 & 50 & & \\
\hline
\end{tabular}

\subsection{Test result analysis}

Tables 4 and 5 show that the primary and secondary orders that influence the missing seed rate were $\mathrm{A}>\mathrm{C}>\mathrm{B}$, the optimal combination was $\mathrm{A}_{2} \mathrm{C}_{3} \mathrm{~B}_{2}$. The primary and secondary order influencing 1-3 seeds per hole were $\mathrm{A}>\mathrm{C}>\mathrm{B}$, the optimal combination was $\mathrm{A}_{2} \mathrm{C}_{2} \mathrm{~B}_{3}$. The primary and secondary order influencing $\geq 4$ seeds per hole were $\mathrm{C}>\mathrm{A}>\mathrm{B}$, the optimal combination was $\mathrm{C}_{1} \mathrm{~A}_{4} \mathrm{~B}_{2}$.

\subsection{Verification test}

Table 4 and 5 show that the $\mathrm{A}_{2} \mathrm{C}_{2} \mathrm{~B}_{3}$ combination is prior to improve the qualified rate of 1-3 seeds per hole. With a combination of $\mathrm{A}_{2} \mathrm{C}_{2} \mathrm{~B}_{3}$, a rotational speed of $20 \mathrm{r} / \mathrm{min}$, a vacuum of $2 \mathrm{kPa}$, and a $2.0 \mathrm{~mm}$ straight hole, confirmatory tests for gathering statistics for 750 holes were performed and recorded. The results are listed in Table 6 which shows that the missed seed per hill rate is $2.0 \%$, the $1-3$ seeds per hole qualified rate is $95.3 \%$, the $\geq 4$ over-sowing rate is $2.7 \%$. These values satisfy the requirements for precision hybrid rice seeding. 
Table 4 Experimental plan and test results

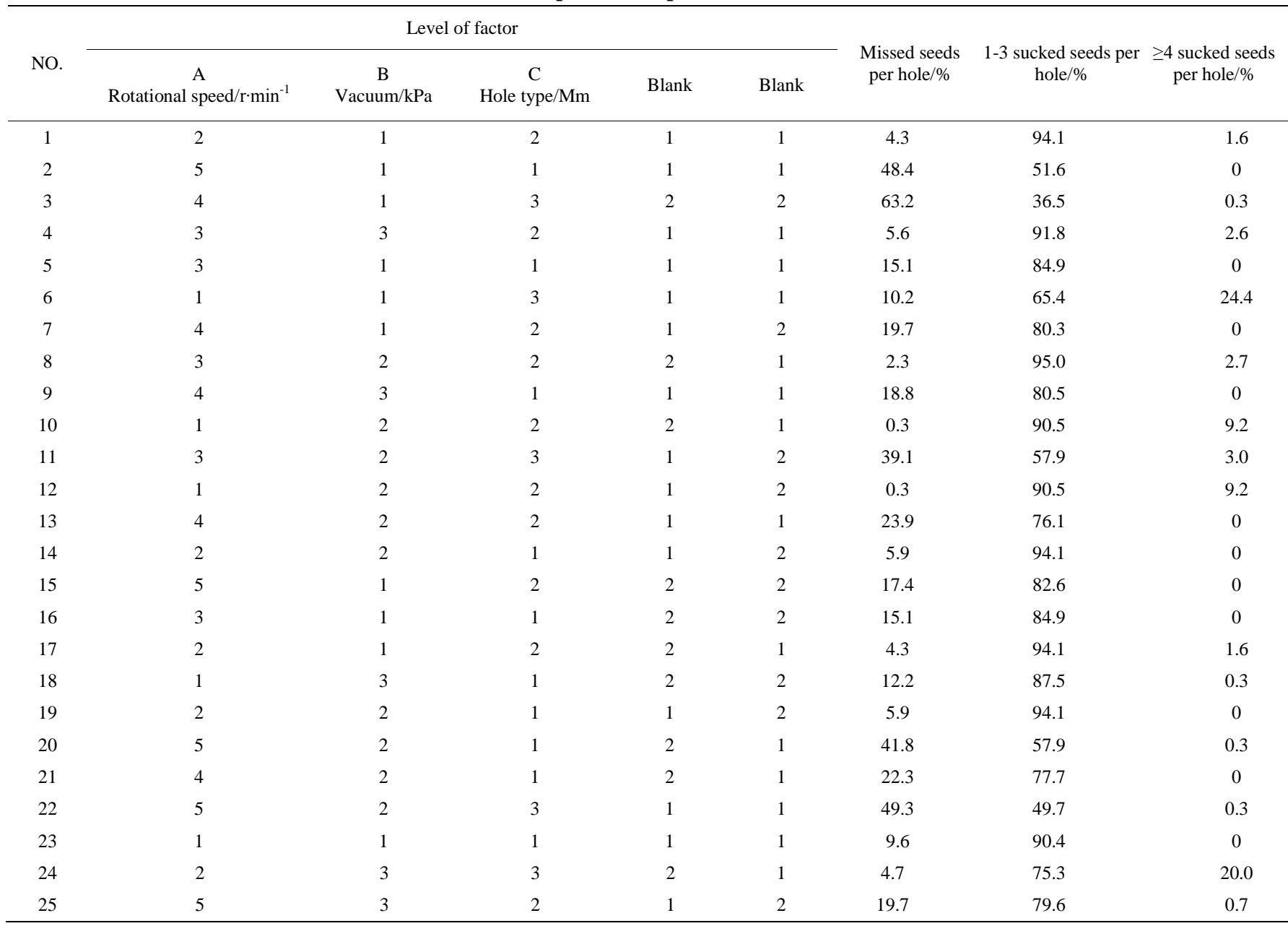

Table 5 Intuitive analysis of rice seeds for cylinder seeder

\begin{tabular}{|c|c|c|c|c|c|c|c|c|c|}
\hline \multirow[b]{2}{*}{$\begin{array}{l}\text { Analysis } \\
\text { item }\end{array}$} & \multicolumn{3}{|c|}{ Missed seeds per hole } & \multicolumn{3}{|c|}{ 1-3 sucked seeds per hole } & \multicolumn{3}{|c|}{$\geq 4$ sucked seeds per hole } \\
\hline & $\begin{array}{c}\text { A } \\
\text { Rotational speed } \\
/ \mathrm{r} \cdot \mathrm{min}^{-1}\end{array}$ & $\begin{array}{c}\mathrm{B} \\
\text { Vacuum } \\
/ \mathrm{kPa}\end{array}$ & $\begin{array}{c}\text { C } \\
\text { Hole type } \\
\text { /mm }\end{array}$ & $\begin{array}{c}\text { A } \\
\text { Rotational speed } \\
/ \mathrm{r} \cdot \mathrm{min}^{-1}\end{array}$ & $\begin{array}{c}\text { B } \\
\text { Vacuum } \\
/ \mathrm{kPa}\end{array}$ & $\begin{array}{c}\text { C } \\
\text { Hole type } \\
\text { /mm }\end{array}$ & $\begin{array}{c}\text { A } \\
\text { Rotational speed } \\
/ \mathrm{r} \cdot \mathrm{min}^{-1}\end{array}$ & $\begin{array}{c}\mathrm{B} \\
\text { Vacuum } \\
/ \mathrm{kPa}\end{array}$ & $\begin{array}{c}\text { C } \\
\text { Hole type } \\
\text { /mm }\end{array}$ \\
\hline $\bar{K}_{1}$ & 6.58 & 20.73 & 19.51 & 84.84 & 76.48 & 80.36 & 8.62 & 2.79 & 0.06 \\
\hline $\bar{K}_{2}$ & 5.02 & 18.88 & 9.55 & 90.34 & 78.35 & 87.46 & 4.64 & 2.70 & 2.99 \\
\hline $\bar{K}_{3}$ & 14.98 & 12.2 & 33.3 & 82.84 & 82.94 & 56.96 & 2.12 & 4.72 & 9.6 \\
\hline $\bar{K}_{4}$ & 29.58 & & & 70.22 & & & 0.06 & & \\
\hline $\bar{K}_{5}$ & 35.32 & & & 64.28 & & & 0.26 & & \\
\hline$R$ & 30.3 & 8.53 & 23.75 & 26.06 & 6.46 & 23.4 & 8.56 & 2.02 & 9.54 \\
\hline \multirow{2}{*}{$\begin{array}{l}\text { Optimal } \\
\text { combination }\end{array}$} & A & $\mathrm{C}$ & B & A & $\mathrm{C}$ & B & $\mathrm{C}$ & A & B \\
\hline & \multicolumn{3}{|c|}{$\mathrm{A}_{2} \mathrm{C}_{3} \mathrm{~B}_{2}$} & \multicolumn{3}{|c|}{$\mathrm{A}_{2} \mathrm{C}_{2} \mathrm{~B}_{3}$} & \multicolumn{3}{|c|}{$\mathrm{C}_{1} \mathrm{~A}_{4} \mathrm{~B}_{2}$} \\
\hline
\end{tabular}

\section{Field tests}

\subsection{Test conditions and methods}

The field tests were carried out at the South China Agricultural University Cencun teaching and scientific farm in Aug 2017. The area of the plots was about two acres. The plots were soaked, rotary-tilled, levelled, and precipitated prior to the field tests. The test seeds were the Indica two-line hybrid rice variety Jingliangyou 1212, which has a growth period of $116.9 \mathrm{~d}$. The sowing machine was a hill-drop pneumatic central cylinder direct-seeding machine for hybrid rice, and the row spacing was $200 \mathrm{~mm}$. The field tests are illustrated in Figure 8.

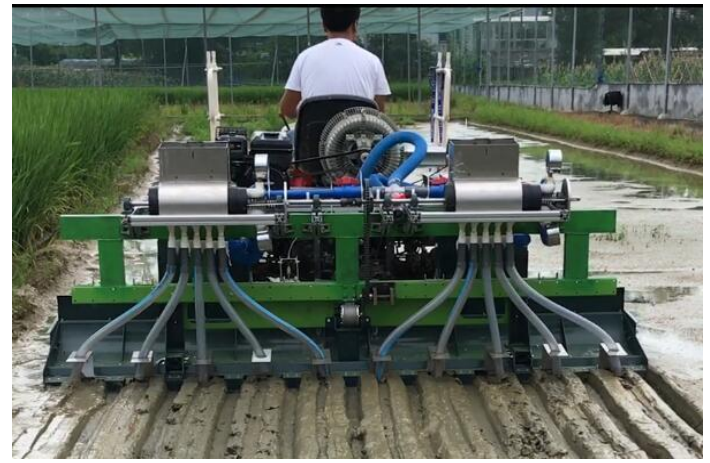

Figure 8 Direct-seeding machine in paddy field 


\subsection{Results of seeding accuracy}

Based on the test results listed in Tables 3-5 and the state of the field, the test conditions were set at about $0.5 \mathrm{~m} / \mathrm{s}$ of machine speed, $2 \mathrm{kPa}$ negative pressure of the metering device, $2 \mathrm{~mm}$ straight holes, $1.5 \mathrm{kPa}$ of blowing positive pressure, $3 \mathrm{~cm}$ sowing furrow depth, and $6 \mathrm{~cm}$ ditch depth. The transmission ratio of the PTO to the seeder was 9:1. According to the GB/T 6973-2005 ${ }^{[34,35]}$ single (precise) test method, every seed row was recorded for 250 hills and repeated three times. Seed amount of 1-3 is qualified, $<1$ is deemed missed seeding, and $>3$ is deemed over-sowing. The test results show that under the test conditions, the missed seed per hole rate is $2.7 \%$, the $1-3$ seeds per hole qualified rate is $91.6 \%$, and the $\geq 4$ over-sowing rate is $5.7 \%$.

\subsection{Results of final product}

The test was arranged in Nov 2017, four experimental plots with $1 \mathrm{~m} \times 1 \mathrm{~m}$ (as: 1 to 4 plots) were randomly selected in sowing

area, and the rice ears within that area were clipped into corresponding net bags, which were then allowed to air dry until the water content was about $13.5 \%$.

Data extraction was performed gather data on the average efficient panicle, the seed-setting rate, the 1000-grain weight, and the theoretical yield of the experimental plots. The field production data are listed in Table 6 . The growth of the rice and the seedling emergence in the field are shown in Figure 9.

Table 6 Field production data

\begin{tabular}{lccccc}
\hline \multicolumn{1}{c}{ Item } & 1 & 2 & 3 & 4 & Average \\
\hline Efficient panicle A/ears & 214 & 241 & 214 & 256 & 231.25 \\
Seed-setting rate B/\% & 90.63 & 90.36 & 90.52 & 88.82 & 90.08 \\
1000-grain weight C/g & 22.10 & 21.80 & 22.30 & 23.60 & 22.450 \\
Theoretical yield D $/ \mathrm{kg} \cdot \mathrm{hm}^{-2}$ & 6867.30 & 6395.40 & 6744.30 & 8415.45 & 7107.90 \\
\hline
\end{tabular}

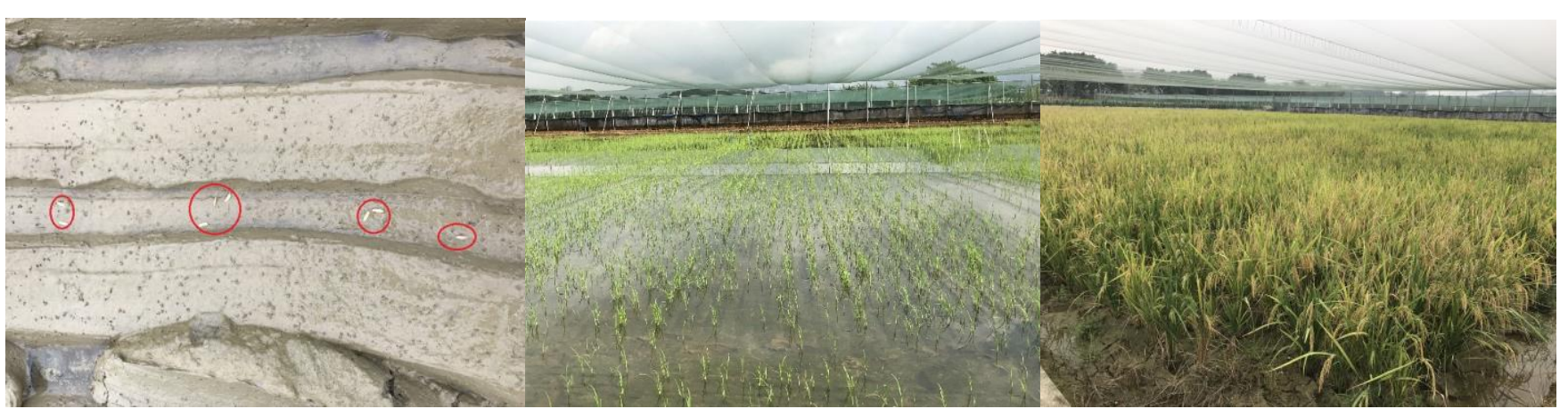

Figure 9 Field seeding (red circles indicate seeds)

\section{Conclusions}

1) A pneumatic central-cylinder device including double cylinders, a ventilation chamber shell, and bilateral cleaning devices was designed. The hole type, rotational speed of the cylinder and cavity vacuum degree were optimized to maximize the performance of the seed-metering device. The orthogonal experiments results showed that when the rotational speed of the cylinder was $30 \mathrm{r} / \mathrm{min}$, the cavity vacuum degree was $2 \mathrm{kPa}$, and the hole diameter was $2 \mathrm{~mm}$ (straight hole), the $(<1)$ missed seed per hole percentage is $2.3 \%$, the (1-3) (qualified rate) percentage is $95 \%$, the (>4) (over sowing) rate is $2.7 \%$.

2) A hill-drop pneumatic central cylinder direct-seeding machine for hybrid rice was designed, included a riding rice transplanter with a four-wheel-drive planter chassis, a synchronous ditching and ridging chassis, a double pneumatic central-cylinder device, a vortex pump and piping tubes. The results of the field testing showed that the missed seed per hole percentage was $2.7 \%$, the qualified rate (1-3) percentage was $91.6 \%$, and the over-sowing $(\geq 4)$ percentage was $5.7 \%$.

3) The hill-drop pneumatic central cylinder direct-seeding machine for hybrid rice can sow ten rows at a time with two seeders, which can reduce the need of power consumption and air pressure.

4) The yield test showed that the average effective panicle was 231.25 ears, the average seed setting rate was $90.08 \%$, the average 1000-grain weight was $22.45 \mathrm{~g}$, and the average yield was $7107.9 \mathrm{~kg} / \mathrm{hm}^{2}$, which is about $26.14 \%$ higher than the average rice yield $\left(5634.9 \mathrm{~kg} / \mathrm{hm}^{2}\right)$ of Guangdong province in $2016^{[36]}$.

In present study, a hill-drop pneumatic central cylinder direct-seeding machine for hybrid rice was designed and studied. However, the vibration frequency and amplitude of the seed box, the cleaning device installation parameters, and the air-blowing pipeline distribution parameters required in-depth study in the future.

\section{Acknowledgements}

The authors acknowledge the funding from the Introduction of the National Key Research and Development Program of China (2018YFD0100800), the earmarked fund for China Agriculture Research System(CARS-01-41), International Advanced Agricultural Science and Technology Program (948 plan) (Project No.2016-X24), National Postdoctoral Program for Innovative Talents (BX201700083).

\section{[References]}

[1] Ma G H, Yuan L P. Hybrid rice achievements, development and prospect in China. Journal of Integrative Agriculture, 2015; 14(2): 197-205.

[2] National Bureau of Statistics of the People's Republic of China.Annual data, Rice sown area. http://data.stats.gov.cn/ easyquery.htm?cn=C01.

[3] Hu Z X, Tian Y, Xu Q S. Review of extension and analysis on current status of hybrid rice in China. Hybrid Rice, 2016; 31(2): 1-8.

[4] Luo X W, Jiang E C, Wang Z M. Precision rice hill-drop drilling machine. Transactions of the CSAE, 2008; 24(12): 52-56. (in Chinese)

[5] Zeng S, Tang H T, Luo X W. Design and experiment of precision rice hill-drop drilling machine for dry land with synchronous fertilizing. Transactions of the CSAE, 2012; 28(20): 12-19. (in Chinese)

[6] Singh R S, Gite L P. Technological change in paddy production: A comparative analysis of traditional and direct seeding methods of cultivation. AMA-Agricultural mechanization in Asia Africa and Latin America, 2012; 43(3): 41-46.

[7] Bhatt R, Kukal S S. Direct seeded rice in South Asia. Sustainable Agriculture Reviews, 2015; 18: 217-252.

[8] Dixit A, Manes G S, Singh A, et al. Evaluation of direct-seeded rice drill against Japanese manual transplanter for higher productivity in rice. Indian Journal of Agricultural Sciences, 2010; 80(10): 884-887.

[9] Zhang G Z, Zang Y, Luo X W, Wang Z M, Zhang Q, Zhang S S. Design and indoor simulated experiment of pneumatic rice seed metering device. 
Int J Agric \& Biol Eng, 2015; 8(4): 10-18

[10] Zhang G Z, Zang Y, Luo X W. Line-churning tooth design and metering accuracy experiment of rice pneumatic precision hill-drop seed metering device on pregnant Japonica rice seed. Transactions of the CSAE, 2014; 30(17): 1-9. (in Chinese)

[11] Xing H, Wang Z M, Luo X W, Cao X M, Liu C B, Zang Y, General structure design and field experiment of pneumatic rice direct-seeder. Int J Agric \& Biol Eng, 2017; 10(6): 31-42

[12] Xing H, Zang Y, Wang Z M. Design and experiment of stratified seed-filling room on rice pneumatic metering device. Transactions of the CSAE, 2015; 31(4): 42-48. (in Chinese)

[13] Zhang S, Xia J F, Zhou Y. Design and experiment of pneumatic cylinder-type precision direct seed-metering device for rice. Transactions of the CSAE, 2015; 31(1): 11-19.

[14] Fu Y M, Wu G, Lv Y. Design and experiment of pneumatic cylinder-type precision seed-metering device. Journal of Agricultural Mechanization Research, 2016; 38(1): 172-176. (in Chinese)

[15] Zhao Z, Li Y M, Chen J. Dynamic analysis on seeds pick-up process for vacuum-cylinder seeder. Transactions of the CSAE, 2011; 27(7): 112-116. (in Chinese)

[16] Zuo Y J, Ma X, Qi L. Seeding experiments of suction cylinder-seeder with socket-slot. Transactions of the CSAE, 2010; 26(11): 141-144. (in Chinese)

[17] Zuo Y J, Ma X, Yu D L. Flow field numerical simulation of suction cylinder-seeder for rice bud seed with socket-slot. Transactions of the CSAM, 2011; 42(2): 58-62. (in Chinese)

[18] Gao X J, Zhou J H, Lai Q H. Design and experiment of pneumatic cylinder precision seed-metering device for panax notoginseng. Transactions of the CSAE, 2016; 32(2): 20-28. (in Chinese)

[19] Liao Y T, Wang L, Liao Q X. Design and test of an inside-filling pneumatic precision centralized seed-metering device for rapeseed. Int $\mathbf{J}$ Agric \& Biol Eng, 2015; 8(4): 10-18.

[20] Yu J J, Liao Y T, Cong J J. Yang S, Liao Q X, Simulation analysis and match experiment on negative and positive pressures of pneumatic precision metering device for rapeseed. Int J Agric \& Biol Eng, 2011; 7(3): $1-11$.

[21] Karayela D, Wiesehoffb M, Özmerzia A, et al. Laboratory measurement of seed drill hill spacing. and velocity of fall of seeds using high-speed camera system. Computers and Electronics In Agriculture, 2006; 50: 89-96.

[22] Singh R C, Singh G, Saraswat D C. Optimisation of design and operational parameters of a pneumatic seed metering device for planting cotton seeds. Biosystems Engineering, 2005; 92(4): 429-438.

[23] Shao X, Hu J, Huang Y. DEM simulation and analysis of seeds supply by the vibrating seed box of magnetic cylinder seeder. International Conference on Computer and Computing Technologies in Agriculture. Springer Berlin Heidelberg, 2010; pp.401-408.

[24] Kumar G V P, Srivastava B, Nagesh D S. Modeling and optimization of parameters of flow rate of paddy rice grains through the horizontal rotating cylindrical cylinder of cylinder seeder. Computers and Electronics in Agriculture, 2009; 65(1): 26-35.

[25] Zhan Z, Li Y M, Jin C. Numerical analysis and laboratory testing of hill spacing. uniformity performance for vacuum- cylinder precision seeder. Biosystems engineering, 2010; 106(4): 344-351.

[26] Atnayake R M C, Balasoriya B M C P. Re-Design, fabrication, and performance evaluation of manual conical cylinder seeder: A case study. Applied Engineering in Agriculture, 2013; 29(2): 139-147.

[27] Wang Y J, Zhang Z L, Jiang Y Z. Based on the CFD technologys negative pressure chamber air flow field simulation analysis of the aspiration cylinder precision seeding mechanism. Advanced Materials Research, 2013; 706-708: 1226-1230.

[28] Pang C L, Cai Z M, Su C Y, Huang J L. Design and experimental study on air-suction two-layer cylinder rice seeder. Transactions of the CSAE 2000; 16(5): 52-55. (in Chinese)

[29] Zhang B P. Design principle of seeding machine. Beijing: China Machine Press, 1992. (in Chinese)

[30] Ratnayake R M C , Balasoriya B M C P. Re-Design, fabrication, and performance evaluation of manual conical cylinder seeder: A case study. Applied Engineering in Agriculture, 2013; 29(2): 139-147.

[31] Deng W J, Li Z W, Qiu X L,Wang W, Wu X, Zheng D K. The research of rice liquidity and arching based on crossed diversion seed-box. Journal of Agricultural Mechanization Research, 2013; 12: 145-149.

[32] Chen K J, Xu W L. Effect of moisture content on mechanical properties of rice. Transactions of the CSAM, 2005; 11: 177-178. (in Chinese)

[33] Cengel Y A, Cimbala J M. Fluid mechanics fundamentals and applications. McGraw-Hill Higher Education, 2013.

[34] GB/T 6973-2005 single (precise) test method. National Standards of the People's Republic of China. (in Chinese)

[35] Li Y Y, Hu C R. Experimental design and data processing. Beijing: Chemical Industry Press, 2008. (in Chinese)

[36] National Bureau of Statistics of China. Statistical Yearbook of China (2015). Beijing: China Statistics Press, 2015: 231-239. (in Chinese) 